\title{
Personalizing the theme park: Psychometric profiling and physiological monitoring
}

\author{
Stefan Rennick-Egglestone ${ }^{1}$, Amanda Whitbrook ${ }^{2}$, Caroline Leygue ${ }^{3}$, \\ Julie Greensmith ${ }^{1}$, Brendan Walker ${ }^{4}$, Steve Benford ${ }^{1}$, Holger Schnädelbach ${ }^{1}$, \\ Stuart Reeves ${ }^{3}$, Joe Marshall ${ }^{1}$, David Kirk ${ }^{1}$, Paul Tennent ${ }^{1}$, \\ Ainoje Irune ${ }^{1}$ and Duncan Rowland ${ }^{5}$ \\ ${ }^{1}$ School of Computer Science, University of Nottingham, Jubilee Campus, \\ Wollaton Road, Nottingham, NG8 1BB \\ \{sre,jqg,sdb,hms,jqm,dsk,pxt,aai\}@es.nott.ac.uk \\ ${ }^{2}$ BAE Systems, Systems Engineering Innovation Centre, University of Loughborough, \\ Holywell Park, Loughborough, LE11 3TU \\ a.m.whitbrook@lboro.ac.uk \\ ${ }^{3}$ Horizon Digital Economy Research, Sir Colin Campbell Building, \\ University of Nottingham Innovation Park, Triumph Road, Nottingham, NG7 2TU \\ ${ }^{4}$ Aerial, 258 Globe Road, London, E2 0JD \\ info@aerial.fm \\ ${ }^{5}$ School of Computer Science, University of Lincoln, Brayford Pool, Lincoln, LN6 7TS \\ drowland@lincoln.ac.uk
}

\begin{abstract}
Theme parks are important and complex forms of entertainment, with a broad user-base, and with a substantial economic impact. In this paper, we present a case study of an existing theme park, and use this to motivate two research challenges in relation to user-modeling and personalization in this environment: developing recommender systems to support theme park visits, and developing rides that are personalized to the users who take part in them. We then provide an analysis, drawn from a real-world study on an existing ride, which illustrates the efficacy of psychometric profiling and physiological monitoring in relation to these challenges. We conclude by discussing further research work that could be carried out within the theme park, but motivate this research by considering the broader contribution to user-modeling issues that it could make. As such, we present the theme park as a microcosm which is amenable to research, but which is relevant in a much broader setting.
\end{abstract}

Keywords: Psychometrics, physiological monitoring, theme park.

\section{Introduction}

Leisure and entertainment is a topic of interest for researchers involved in usermodeling and personalization. One application is e-commerce systems such as Amazon or eBay. These provide personalized, collaborative recommendations for leisure items such as books, DVDs and computer games, generated through an analysis of on-line activities [1]. Other applications include the personalized 
recommendation of leisure activities to partake in whilst on holiday [2], or the recommendations of playlists of music [3]. Working within a framework of affective computing [4], there have been attempts to model human emotions such as frustration [5], based on data collected from sensors. Prototypes of games that detect and respond to these emotions have then been built, using a variety of sensing devices and prosthetics to gather data (for example, [6]).

Approaching the theme of leisure and entertainment from a novel direction, this paper motivates the application of personalization technologies to theme parks, which are highly complex entertainment spaces that contain a wide variety of different forms of attraction. As a whole, the theme park industry caters for hundreds of millions of visitors every year [7], and theme park operators have significant budgets to spend on novel forms of entertainment. Although the theme park provides enormous scope for computational interventions, there are few examples of published research in the theme park. An exception is [8], and the authors have also developed a number of publications around the theme park. So far, these have focused on issues such as building novel interfaces for spectators [9], and the prototyping of new forms of ride $[10,11]$. An initial investigation into the use of user-profiling technologies in the theme park has also been published [12].

Building on this research program, this paper makes a contribution to research through a detailed exploration of the personalization of theme park experiences for visitors. It begins by providing a case-study of a typical theme park environment, which is used to highlight the potential benefits to visitors and to park operators that can be provided user-modeling and personalization technologies. This section is used to introduce two themes of research which the authors are interested in - namely the development of recommender systems for the theme park environment, and the development of novel forms of ride that are capable of responding to those that ride them. The section outlines a set of research challenges related to the themes of usermodeling and personalization. It then provides an overview of research which the authors have conducted in this space.

Following on from this section, this paper presents two analyses of a novel corpus of data which was collected in the theme park, through a study involving 56 participants who took part in a single ride. This data contains a detailed profile of these participants, which include assessments against two standard psychometric personality tests - the Big Five and the Sensation Seeking Scale, which are introduced later in this paper. It also includes a series of physiological measurements which were captured through wearable computing technologies. Analysis presented in this paper is then used to highlight the predictive power of psychometric profiles for experience on the ride, suggesting the inclusion of psychometric measures in a profile of theme park visitors which could be used by a recommender system. It is also used to demonstrate the efficacy of using physiological data to measure experience on a ride, suggesting the use of physiological measurements to personalize ride experiences.

Finally, after having presented these analyses, the paper concludes by discussing further work required to make the personalization of theme park experiences a reality. It also considers the broader contributions to knowledge that can be made through research in the theme park, therefore emphasizing its wider applicability. 


\section{The theme park environment}

Theme parks are a popular form of entertainment around the world, and their design differs substantially. To provide a solid grounding for the remainder of this paper, this section now provides a case-study of a particular theme park with which the authors have interacted. Details presented in this case-study have been drawn from interviews with park management and other staff. Having presented this case-study, we then highlight the potential interest of the theme park environment to researchers interested in user-modeling and personalization, by setting two research challenges. The remainder of this paper is then structured around these research challenges.

\subsection{An overview of Alton Towers}

Alton Towers [13] is a theme park in the UK. It attracts more than 2 million visitors every year, with a daily capacity of 30,000 visitors. Figure 1 shows the stylized map that is provided to visitors on arrival. This highlights the various attractions at the park. These include a large number of thrilling and family-orientated rides, restaurants, shops, a formal garden (which existed before the theme park) and a hotel complex. Cameras have been installed onto many rides, and shops already provide souvenirs that have been personalized with photographs or video of riders. Alton Towers has a consistent theming, which is known internally as "Fantastical Escapism". This theming begins with "Towers Street", the single entry point to the park, and continues throughout.

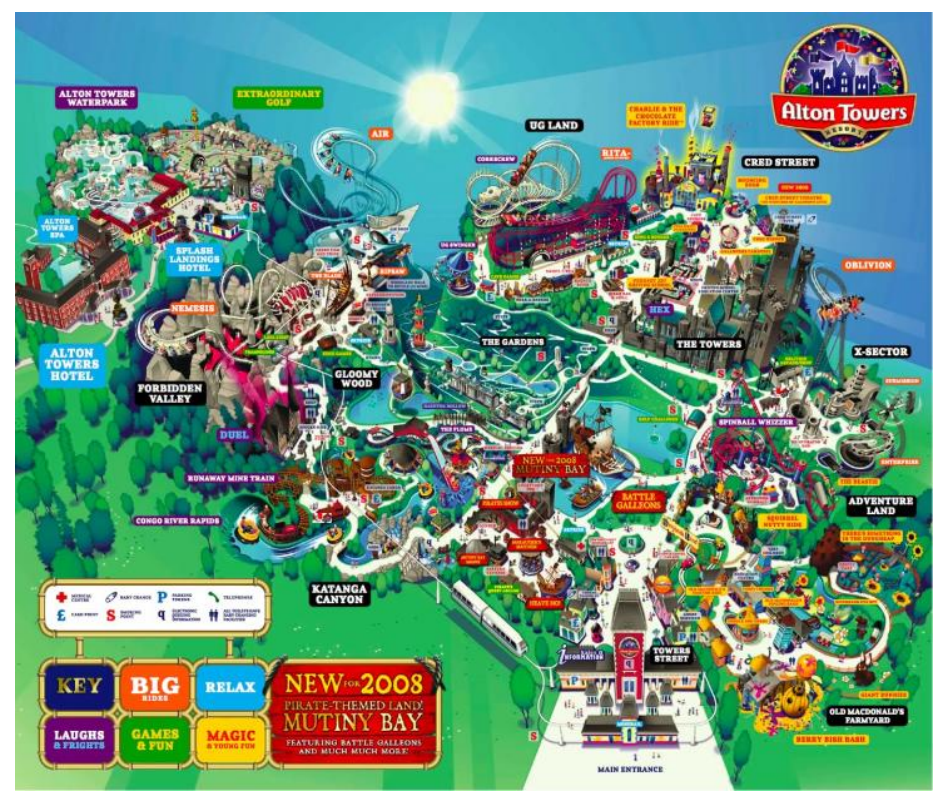

Figure 1 Alton Towers map 
The rides at Alton Towers are the big draw for most visitors, and because of their limited capacity, queues can be large (sometimes longer than an hour for new rides). To reduce queuing, the most popular rides are distributed around the furthest edge of the park, so that crowds have spread out by the time they get there. There are also boards illustrating queuing times for these rides, which are updated manually once an hour. Staff at the park believe that many visitors walk move in an anti-clockwise direction around the park, causing queuing problems at particular locations. Queuing is also worse at peak times, or at particularly new rides.

Additionally, for many visitors, their interaction with a particular park visit begins on-line - through a ticket purchasing system that offers discounts in comparison to the gate price. Visitors can also purchase extra tickets that allow them to jump to the front of queues all day (most expensive option) or on individual rides (cheapest option). On-line information provided by Alton Towers allows visitors to plan their day, and additional information can be found on a variety of fan-sites (for example, [14]). Alton Towers also maintains a presence on Facebook and Twitter.

\subsection{Challenges for user-modeling and personalization in the theme park}

Having provided a short case-study of a particular theme-park, we now outline two research challenges which involve user-modeling and personalization. Section 3 of this paper then presents initial studies that provide knowledge in relation to these.

\section{Challenge one: The theme park recommendation system.}

A day at a theme-park can be an expensive investment for a family, potentially involving travel costs, park entry fees, food and accommodation. Once at the park, there is an enormous selection of entertainment on offer. However, given the time taken to traverse the park, and given the possibility of large queues that has been outlined above, there is a significant potential for a frustrating experience. This suggests the challenge of creating an information system that assists visitors, and which recommends a personalized experience. Such a system might embed aspects of collaborative recommendation [15]. It might also interface with future park systems that monitored visitor movements, with on-line systems for booking tickets, or with information collected during previous visits.

\section{Challenge 2: Personalized experiences on rides}

Many of the rides at Alton Towers are thrilling, but each provides essentially the same experience to all visitors. However, developments in ride technology provide the potential for personalized ride experiences - for example, some rides now embed CAVE-like motion-platform and projection technologies [16], whilst others provide for individual actuation of seat movements [17]. In addition, technologies such as RFID that allow for the identification of visitors are already in operation at Alton Towers, for the purposes of identifying video sequences that feature particular riders, which are then used to produce souvenirs. This suggests the challenge of developing rides that can be personalized to individual riders, or to groups of riders. Such rides may draw on information collected by on-line systems, or during previous visits. 


\section{Results of studies constructed around these challenges}

Having motivated two research challenges relating to the theme park, we now present two analyses which contribute knowledge in relation to these. Both draw on data collected during a single study by the authors in the theme park, which involved 56 participants. During this study, each had one ride on Oblivion, a major attraction at Alton Towers. Before this ride, a personal profile was collected for each rider, and during the ride, wearable equipment was used to record aspects of their physiological response. Oblivion is constructed around a vertical drop into a tunnel, and a photograph of this drop is shown in figure 2. This figure also shows an abstract map of the ride, which has been labeled with 10 key points. Immediately after the ride, participants used paper forms to provide a numerical assessment of their emotional state at each of these points against the circumplex model [18], which is defined by two dimensions, arousal and valence. This model is commonly used in research requiring the quantification of emotion, and seems particularly relevant to intense experiences such as theme park rides. In the context of this study, arousal was defined on a scale of 0 to 10 as being how much the rider felt "alert, with your body pumped up and buzzing, ready for action", whilst valence was defined on a scale of -5 to +5 as being how much the rider felt "positive or good" or "negative or bad". Data collected against this model has been used in both analyses presented below.
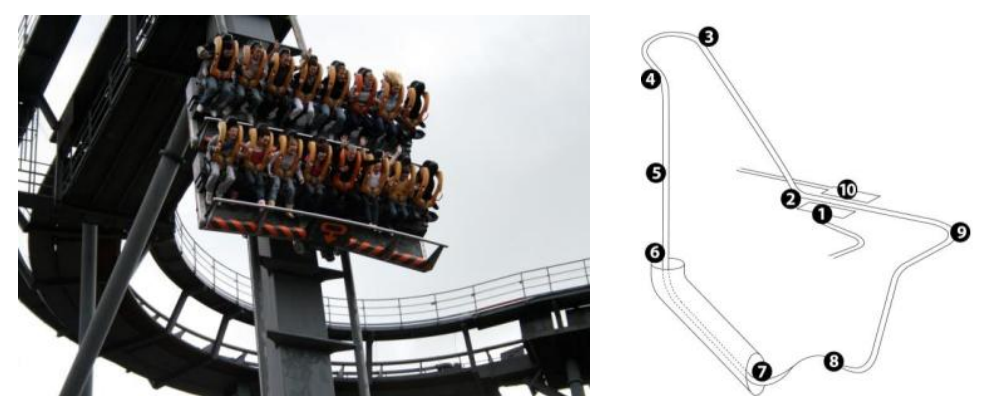

Figure 2 Beginning of vertical drop (left) and map of ride (right): 1: Loading bay 2-3: Lift hill 4-5: Drop 6-7: Tunnel 8: Curves 9-10: End of ride

\subsection{Analysis one: relationships between personal profiles and ride experience}

A key component of a system constructed in response to challenge one (the theme park recommendation system) could be a module that uses a profile of a user to recommend a series of rides that they will enjoy, and which assimilates geographic information about the layout of rides and the size of queues. For users who have visited parks before, such a profile may draw on records of previous visits. However, an alternative approach is required for first-time visitors. For this group, we have been investigating the efficacy of including demographic and psychometric personality data in a visitor profile. Whilst the use of demographics in profiling is well-accepted, the use of personality data in personal profiles for recommender systems is a current 
topic of user-modeling research [19], where it is appropriate for the recommendation of experiences that are mediated by personality. In this section, we provide proof-ofconcept evidence that this is the case in the theme park.

Our approach in gathering this evidence has been to use data collected during the Oblivion study, allowing us to investigate relationships between demographic and psychometric dimensions and self-reported experience on a single ride. Psychometric personality profiling requires users to fill out a questionnaire, from which numeric scores on a set of pre-determined personality dimensions are produced. Informed by discussions with psychology colleagues, we chose two commonly-used psychometric personality profiling tools for this study: the Big Five [20], a general-purpose test, and the Sensation Seeking Scale [21], a test designed for investigations into thrilling experiences. Table 1 below summarizes dimensions in our profile. When working with this data, we have used correlation analysis to identify a candidate set of dimensions with significant relationships to self-reported experience. We have then used this candidate set to cluster participants into groups, and tested for significant differences in self-reported experience between groups.

Table 1 Profile (ride count $=$ number of previous rides on Oblivion)

\begin{tabular}{|l|l|l|}
\hline Demographics & Psychometrics - Big 5 & Psychometrics - SSS \\
\hline Age & Openness & Thrill seeking \\
\hline Gender & Conscientiousness & Experience seeking \\
\hline ride count & Extraversion & Disinhibition \\
\hline & Agreeableness & Boredom susceptibility \\
\hline & Neuroticism & \\
\hline
\end{tabular}

To implement the method described above, we first tested for normality (using Shapiro-Wilks). This failed to provide evidence for normality for almost all profiling dimensions in table 1, so we chose to use the Spearman rank correlation, a nonparametric correlation co-efficient, to search for relationships between dimensions shown in table 1 and self-reports of emotion. To add depth to our analysis, we grouped some points in the map shown in figure 1, to generate a set of categories shown in table 2. Only those correlations between personality dimensions and these categories that are significant at a confidence level of $p=0.001$ are then shown in table 3 . The choice of this more stringent confidence level (than the more commonly-used level of $p=0.05$ ) reduces the possibility of seemingly significant correlations occurring by chance when calculating such a large number of correlations.

Based on these correlations, ride count, thrill seeking, extraversion and openness were chosen as candidate dimensions for a future profile. A further investigation was then carried out using the k-means clustering algorithm as implemented by SPSS. After an exploratory analysis, three useful methods of clustering participants were identified (clustering on ride count alone, clustering on thrill seeking alone, and clustering on extraversion and openness together). An analysis of the self-reports of experience provided by members of these groups has demonstrated that there is a significant difference between these self-reports (table 4 - Kruskall-Wallis test, $\mathrm{p}=0.05$ ). Collectively, these statistics provide further evidence for the use of these dimensions in a future personal profile for a theme park recommender system. 
Table 2 Category definitions

\begin{tabular}{|l|l|l|l|l|}
\hline Whole ride & Pre-drop & Hanging & Drop & Post-drop \\
\hline $1-10$ & $1-3$ & 4 & $5-7$ & $8-10$ \\
\hline
\end{tabular}

Table 3 Significant correlations between categories and profiling dimensions

\begin{tabular}{|l|c|c|c|c|c|}
\hline & Extraversion & Openness & Thrill seeking & Gender & Ride count \\
\hline whole_ride_arousal & - & 0.12 & -0.14 & - & -0.19 \\
\hline pre_drop_arousal & - & - & -0.24 & - & - \\
\hline hanging_arousal & - & - & - & - & - \\
\hline drop_arousal & - & 0.21 & - & - & -0.31 \\
\hline post_drop_arousal & - & - & - & - & -0.29 \\
\hline whole_ride_valence & 0.24 & 0.11 & - & -0.15 & - \\
\hline pre_drop_valence & 0.30 & - & 0.24 & - & - \\
\hline hanging_valence & - & - & - & - & 0.36 \\
\hline drop_valence & 0.23 & - & - & -0.30 & - \\
\hline post_drop_valence & - & 0.23 & - & - & -0.28 \\
\hline
\end{tabular}

Table 4 p-values showing significant differences in experience between clusters, generated by three different methods. cs1=clustering by ride count, cs2=clustering by thrill seeking, cs3=clustering by extraversion and openness

\begin{tabular}{|l|c|c|c|}
\hline & cs1 & cs2 & cs3 \\
\hline whole_ride_arousal & 0.000 & 0.003 & 0.007 \\
\hline pre_drop_arousal & 0.040 & 0.001 & - \\
\hline hanging_arousal & - & 0.005 & 0.003 \\
\hline drop_arousal & 0.003 & 0.017 & 0.004 \\
\hline post_drop_arousal & 0.003 & - & 0.000 \\
\hline whole_ride_valence & 0.000 & 0.005 & 0.000 \\
\hline pre_drop_valence & - & 0.000 & 0.029 \\
\hline hanging_valence & - & 0.025 & 0.001 \\
\hline drop_valence & 0.001 & - & 0.006 \\
\hline post_drop_valence & 0.009 & - & \\
\hline
\end{tabular}

\subsection{Analysis two: proof-of-concept evidence for heart-rate monitoring}

Challenge 2 involves the design of rides that are personalized to participants. This could simply make use of a profile, gathered in advance, to select from a number of pre-defined ride programs. However, we have been investigating a more challenging form of personalization which involves the ride adapting dynamically to the responses of its participants. This raises the question of what responses to monitor, how to model these responses, and how to use these models in personalization algorithms. Informed by the field of affective computing [4], we have chosen to investigate the potential of using wearable computing equipment to measure heart-rate response on the ride described above. Heart-rate relates directly to physiological arousal, a measure of body's level of preparation for exciting or fearful situations. Building a ride that responds to individual levels of this arousal is a promising approach, and this section provides proof-of-concept evidence for measuring heart-rate on a ride. 
Given our choice of heart-rate, the collection of proof-of-concept evidence for its use in a dynamically-adaptive ride involves a number of questions. Question 1: does the ride actually affect heart-rate sufficiently for it to be a usable measure in this context? Question 2: is there sufficient variability between riders for this measure to be useful in personalization of experience? Question 3: are there any relationships between heart-rate and emotional experience? In the remainder of this section, we present evidence in relation to all three of these questions.

Figure 3 begins to address the first two, through a plot that shows the distribution of all the individual heart-rates values that were recorded from participants, during six phases of the experience of taking part in the ride. This plot shows an ascending pattern for the sample median throughout most of this experience, with a local maximum whilst waiting to board the ride. The Wilcoxan Rank Sum test was used (at $\mathrm{p}=0.05$ ) to compare the distribution of heart-rate between these boxes, and shows a significant difference between all. In addition, the plot suggests a significant amount of variation in heart-rate across the sample. Box 2, for example, contains some outliers with heart-rates around 60 beats per minute (BPM), and some outliers with heart-rates around $160 \mathrm{BPM}$, and also has a large inter-quartile range. A careful analysis of heart-rate data traces has convinced us that these outliers represent true heart-rates, rather than the results of equipment failures such as drop-outs.

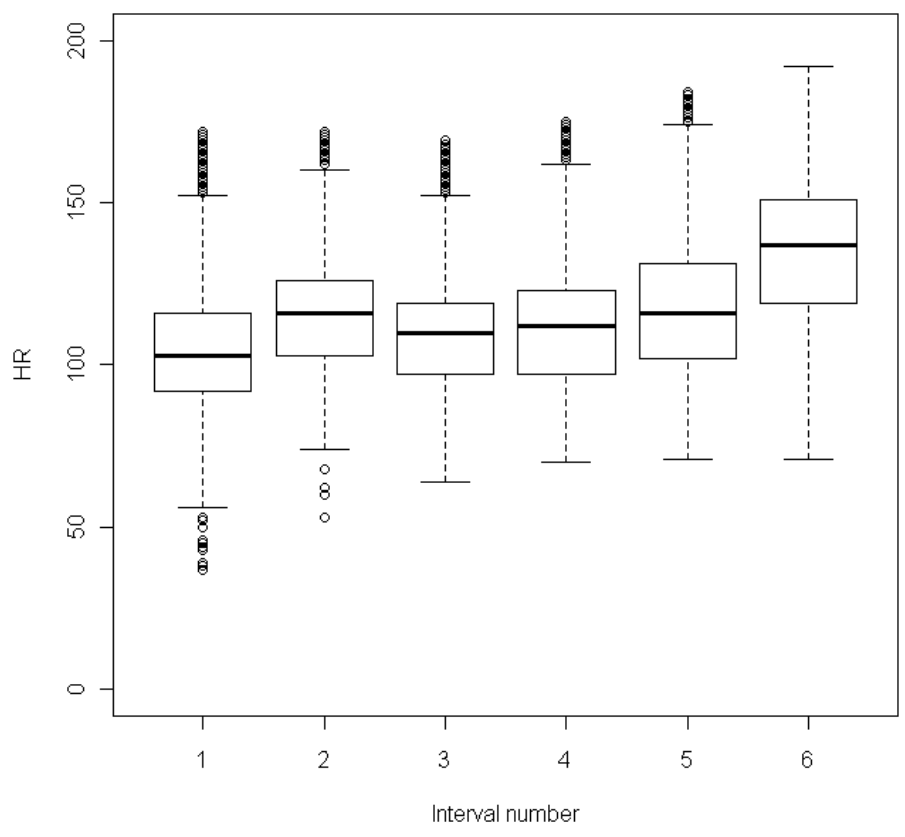

Figure 3 Distribution of individual heart-rate values (BPM)

1: arrival at loading bay 2: waiting to board ride 3: waiting for ride to move 4: ascent of lift hill 5: progress around top 6: drop and remainder of ride 

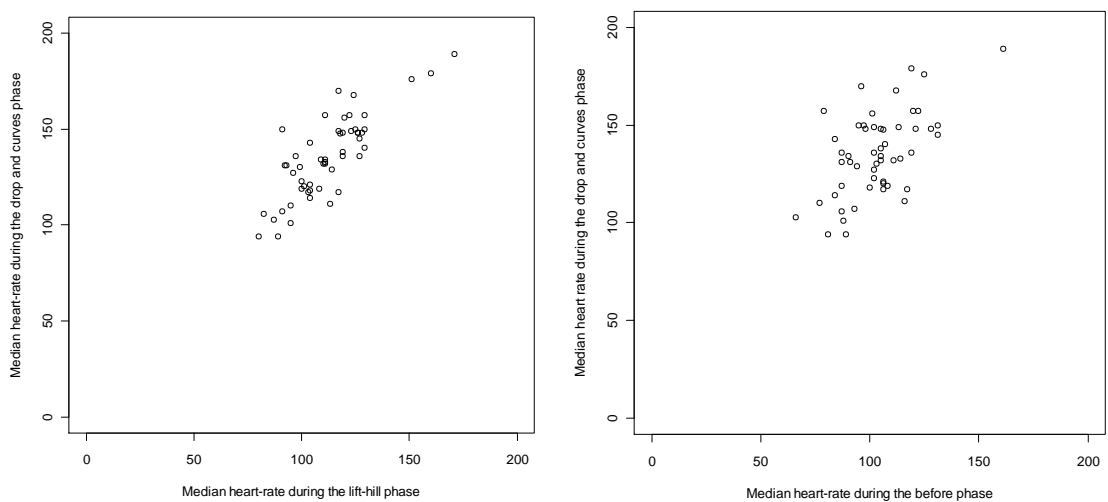

Figure 4 Scatter plots showing distribution of median heart-rates.

Left: ascent of lift-hill ( $x$-axis) against drop and remainder of ride ( $y$-axis) Right: arrival at loading bay (x-axis) against drop and remainder (y-axis)

Further evidence for variation across the sample is provided in the scatter plots shown in figure 4, which compare median heart-rate for participants in different phases of the ride. These show an extreme variation in levels of physiological arousal, far higher than what would be expected in normal life (for which a heart-rate between 60 and 100 BPM is more usual). In addition, they suggest a linear relationship between median heart-rate early in the experience and median heart-rate later in the experience. A regression analysis, at a confidence level of $p=0.05$, confirms the predictive nature of heart-rate both on arrival and during the lift-hill for heart-rate during the drop and the remainder of the ride. Therefore, although the ride clearly affects heart-rate, and although there is sufficient variation in the sample to suggest the value of measuring it, there is more evidence for the measurement of heart-rate before the ride begins, as an indicator of physiological arousal, rather than measuring it whilst the ride is moving. This may reflect the fact that many riders are already fearful or excited before the ride begins, and who are therefore more likely to be fearful or excited throughout the remainder of their experience.

Finally, in relation to question 3, an analysis which involves the use of correlation to search for linear relationships between median heart-rate in the different phases and self-reports of emotional experience has produced mixed results. No significant correlations were found for the first five phases defined in figure 3 above. However, for the final phase, a significant positive correlation was found between self-reported arousal and heart-rate, and a significant negative correlation was found between valence and heart-rate. This phase was then split into four intervals, each of five seconds in duration. The following significant correlations were obtained:

Table 5 Significant correlations during the final phase of the ride

\begin{tabular}{|l|c|c|}
\hline Time in final phase & Correlation with arousal & Correlation with valence \\
\hline $0-5$ seconds & - & 0.30 \\
\hline $5-10$ seconds & 0.29 & 0.34 \\
\hline $10-15$ seconds & 0.39 & 0.31 \\
\hline $15-20$ seconds & 0.36 & - \\
\hline
\end{tabular}




\section{Discussion}

In this paper, we have presented analyses of data collected during a single study, and related them to research challenges in the theme park environment. The first analysis provides proof-of-concept evidence for the use of psychometric personality testing in profiles designed for a theme park recommender system, based on its ability to predict experience on a single ride. The second provides evidence for the use of heart-rate as a profiling tool in the queue of a ride, with the potential to identify participants who are relaxed or highly aroused. As an initial piece of research in this novel application domain, these analyses have raised a significant number of research questions, whose investigation could provide knowledge to support the implementation of real themepark systems in the future. In this final section, we present a selection of these questions, and then conclude by discussing their relevance to user modeling research.

\subsection{Extending the analysis to multiple rides}

A limitation of the initial studies presented in this paper is that they only consider data in relation to a single ride, and interesting research questions might be constructed around their extension to multiple rides. For example, it would be interesting to know whether particular dimensions in our chosen personality tests had particularly strong relationships with experience on different rides, and whether there are other personality tests that are useful in this context. Equally, it would be interesting to know whether heart-rate in the queue is always a predictor for heart-rate later in other rides, or whether variables such as fatigue are more important in some situations.

\subsection{Considering groups in the design of future systems}

Analyses presented in this paper have focused on treating participants individually. However, it is clear from our interviews and observations that many people experience theme park rides in groups (e.g. of family or friends). A recommender system for a day at the park would need to consider the needs of a group rather than just the needs of individuals that compose it; the design and evaluation of such a system might contribute more generally to group-orientated recommender systems research. Similarly, a system that personalizes a ride to its participants might need to consider all the individuals in a particular carriage, rather than just being able to consider participants on an individual basis. Future research might consider different tactics for modeling groups, and selecting programs of operation for them.

\subsection{Repeat visits}

Analyses presented in this paper have considered a single ride on Oblivion. However, interviews with park management suggest a high number of repeat visits, raising the question of how to construct profiles that build over time, and which therefore provide a stronger basis for recommendation or adaption. For example, a user-profile 
might combine psychometric and demographic profiling with records of physiological monitoring or self-report. Such a profile could use identification mechanisms such as RFID, or even be integrated with on-line systems relating to ticket sales. These kinds of profile are a current topic of research, especially given the ever-expanding digital footprint that many people are generating. As such, research work in the theme park might contribute more generally to research in this field, especially given the significant number of visits to theme parks that are made by individuals.

\subsection{Optimization of the use of psychometric personality profiling}

Data in this paper relates to two specific personality tests: the Big Five and the Sensation Seeking Scale. There are a number of interesting issues around the use of these kinds of test that might be considered in future research. In particular, in common with other personality tests, there are a number of different versions of the Sensation Seeking Scale, each of which utilizes a different length questionnaire, and each of which provides a different level of modeling of respondents' personality. There is clearly a trade-off here between the length of time taken to fill out a test and the information that it provides, and such trade-offs may be interesting to investigate in future research. An example of these trade-offs outside of the theme park is present in a number of on-line dating services, such as Match Affinity [23]. Such sites often use quite complex personality tests, and may therefore provide interesting research data for the user-modeling community in relation to their success.

\section{Conclusions}

Most of this paper has focused on the theme park. Two research challenges have been established, and a set of analyses, drawn from a single study, have presented initial knowledge in relation to these. However, the theme park can be seen as a microcosm in which research that is relevant to the wider world can be conducted, potentially aided by theme park infrastructures that support visiting by guests, but which can also provide data for research. Therefore, although research in the theme park is clearly specialized, it has implications that can make a broader contribution, and it is therefore a setting which should be of interest to other researchers.

\section{Acknowledgments}

This work was supported by the Horizon Digital Economy Hub (EP/G065802/1) and by an EPSRC platform award to the Mixed Reality Laboratory (EP/F03038X/1). 


\section{Reference}

1. Schafer, J.B., Konstan, J. and Riedi, J.: Recommender systems in e-commerce. In: $1^{\text {st }}$ ACM conference on electronic commerce. ACM, New York (1999).

2. Duchenaut, N., Partridge, K., Huang, Q., Price, B., Roberts, M., Chi, E.H., Bellotti, V. and Begole, B.: Collaborative filtering is not enough? Experiments with a mixed-model recommender for leisure activities. In: 17th International conference on user-modelling, adaptation and personalization (2009).

3. Hansen, D. and Golbeck, J.: Mixing it up: recommending collections of items. In: $27^{\text {th }}$ annual SIGCHI conference on human factors in computer systems (2009).

4. Picard, R.: Affective Computing. MIT Press, 1997.

5. Klein, J., Moon, Y. and Picard, R.W.: This computer responds to user frustration: Theory, design, and results. Interacting with Computers. 14:2 (2002).

6. Emotiv Epoch interface device, http://www.emotiv.com/

7. Attraction attendance report published by the Theme Entertainment Association, http://www.connectingindustry.com/downloads/pwteaerasupp.pdf

8. Pausch, R., Snoddy, J., Taylor, R., Watson, S. and Haseltine, E.: Disney's Aladdin: first steps towards storytelling virtual reality. In: $23^{\text {rd }}$ international conference on computer graphics and interaction techniques (1996).

9. Schnädelbach, H., Rennick Egglestone, S., Reeves, S., Benford, S. and Walker, B.: Performing Thrill: Designing telemetry systems and spectator interfaces for amusement rides. In: $26^{\text {th }}$ annual SIGCHI conference on human factors in computer systems (2008).

10.Rennick Egglestone, S., Marshall, J., Walker, B., Rowland, D., Benford, S. and Rodden, T.: The Bronco: A proof-of-concept adaptive fairground ride. In: Annual conference on Advances in Computer Entertainment Technology (2009).

11.Marshall, J., Rowland, D., Rennick Egglestone, S., Benford, S., Walker, B. and McAuley, D.: Breath control of amusement rides. In: $29^{\text {th }}$ annual SIGCHI conference on human factors in computer systems (2011).

12.Rennick Egglestone, S., Whitbrook, A., Greensmith, J., Walker, B., Benford, S., Marshall, J., Kirk, D., Schnadelbach, H., Irune, A. and Rowland, D.: Psychometric profiling in the theme park. In: Annual conference on Advances in Computer Entertainment Technology (2010).

13. AltonTowers corporate website, http://www.altontowers.com/

14. Towers Almanac fan-site, http://www.towersalmanac.com/

15. Adomavicius, G. and Tuzhilin, A.: Towards the next generation of recommender systems: a survey of the state-of-the-art and possible extensions. IEEE Transactions on Knowledge and Data Engineering. 17:6 (2005).

16. Charlie and the Chocolate ride at Alton Towers contains a motion platform with projectors, http://www.towersalmanac.com/areas/rides.php?id=85

17. RoboCoaster ride systems, http://www.robocoaster.com

18. Larsen, R.J., Diener, E.: Promises and Problems with the Circumplex Model of Emotion. Review of Personality and Social Psychology, No. 13. (1992).

19. $\mathrm{Hu}, \mathrm{R}$. and $\mathrm{Pu}, \mathrm{P} .:$ A study on user perception of personality-based recommender systems. In: $18^{\text {th }}$ International Conference on user-modelling and personalization (2010).

20. John, O. P., Donahue, E. M., \& Kentle, R. L.: The Big Five Inventory-Versions 4a and 54. Institute of Personality and Social Research, University of California, Berkeley (1991).

21. Zuckerman, M.: Behavioural Expressions and Biosocial Bases of Sensation Seeking. Cambridge University Press (1994).

22. Carlson, N.R.: Physiology of behavior ( $8^{\text {th }}$ edition). Pearson.

23. Match Affinity dating website http://www.matchaffinity.com/ 